\title{
Connectivity of path graphs
}

\author{
Martin Knor $^{1}$ \\ Slovak University of Technology \\ Faculty of Civil Engineering \\ Department of Mathematics \\ Radlinského 11, 81368 Bratislava \\ Slovakia \\ knor@ vox.svf.stuba.sk \\ L'udovít Niepel ${ }^{2}$ and Maryam Malah \\ Department of Mathematics \& Computer Science \\ Faculty of Science \\ Kuwait University \\ P.O. Box 5969 Safat 13060 \\ Kuwait \\ NIEPEL@ MATH-1.sci.kuniv.edu.kw
}

\begin{abstract}
In the paper we present lower bounds for the connectivity of a path graph $P_{2}(G)$ of a graph $G$. Let $\delta \geq 3$ be the minimum degree of $G$. We prove that if $G$ is a connected graph, then $P_{2}(G)$ is at least $(\delta-1)$ connected; and if $G$ is 2-connected, then $P_{2}(G)$ is at least $(2 \delta-2)$ connected. We remark that if $G$ is a $\delta$-regular graph then $P_{2}(G)$ is $(2 \delta-2)$-regular, and hence, if $G$ is 2 -connected then $P_{2}(G)$ is $(2 \delta-2)$ connected; its theoretical maximum.
\end{abstract}

\section{INTRODUCTION AND RESULTS}

Let $G$ be a graph, $k \geq 1$, and let $\mathcal{P}_{k}$ be the set of all paths of length $k$ (i.e., with $k+1$ vertices) in $G$. The vertex set of a path graph $P_{k}(G)$ is the set $\mathcal{P}_{k}$. Two vertices of $P_{k}(G)$ are joined by an edge if and only if the edges in the intersection of the corresponding paths form a path of length $k-1$, and their union forms either a cycle or a path of length $k+1$. This means that the vertices are adjacent if and only if one can be obtained from the other by "shifting" the corresponding paths in $G$.

Path graphs were investigated by Broersma and Hoede in [3] as a natural generalization of line graphs, since $P_{1}(G)$ is the line graph $L(G)$ of $G$. We have to point

\footnotetext{
1) Supported by VEGA grant $1 / 6293 / 99$

2) Supported by Kuwait University grant \#SM 02/00
}

Australasian Journal of Combinatorics 25(2002), pp.175-184 
out that, in the pioneering paper [3] the number $k$ in $P_{k}(G)$ denotes the number of vertices of the paths and not their length. However, in some applications our notation is more consistent, see e.g. [5]. Traversability of $P_{2}$-path graphs is studied in [11], and a characterization of $P_{2}$-path graphs is given in [3] and [9]. Distance properties of path graphs are studied in [2], [6] and [7], and [1] and [10] are devoted to isomorphisms of path graphs.

Let $G$ be a connected graph. It is well-known (and trivial to prove) that $P_{1}(G)$, i.e., the line graph of $G$, is a connected graph. However, this is not the case for $P_{k}$-path graphs if $k \geq 2$. By [7, Theorem 1], we have:

Theorem A. Let $G$ be a connected graph. Then $P_{2}(G)$ is disconnected if and only if $G$ contains two distinct paths $A$ and $B$ of length two, such that the degrees of both endvertices of $A$ are 1 in $G$.

Hence when studying $P_{2}$-path graphs, it seems to be reasonable to restrict ourselves to graphs with minimum degree $\delta \geq 2$. Let $G$ be a graph with a cycle $C$ of even length, $C=a_{1}, a_{2}, \ldots, a_{2 t}$, in which the vertices $a_{2}, a_{4}, \ldots, a_{2 t}$ all have degrees 2. Then $P_{2}(G)$ has a similar cycle, and the connectivity of $P_{2}(G)$ is at most 2. Similarly, the connectivity of $P_{2}^{i}(G), i \geq 2$, is at most 2 (see the definition of iterated path graphs below). However, the situation changes rapidly when the minimum degree of $G$ is at least 3. We prove the following theorem.

Theorem 1. Let $G$ be a connected graph with minimum degree $\delta \geq 3$. Then $P_{2}(G)$ is $(\delta-1)$-connected.

Let $G_{\delta}^{\prime}$ be a connected graph with exactly one vertex of the minimum degree $\delta-1, \delta \geq 3$. Take two copies of $G_{\delta}^{\prime}$, join vertices of the minimum degree by an edge, and denote the resulting graph by $G_{\delta}$. Then it is easy to see that the minimum degree of $G_{\delta}$ is $\delta$, and the connectivity of $P_{2}\left(G_{\delta}\right)$ is at most $\delta-1$. Hence, Theorem 1 is best possible.

For 2-connected graphs, the lower bound of Theorem 1 can be increased to $(2 \delta-2)$.

Theorem 2. Let $G$ be 2-connected graph with minimum degree $\delta \geq 3$. Then $P_{2}(G)$ is $(2 \delta-2)$-connected.

If $G$ is $\delta$-regular graph, then $P_{2}(G)$ is $(2 \delta-2)$-regular. Hence, Theorem 2 is also best possible.

Now we focus on iterated path graphs. These graphs are defined as follows:

$$
P_{k}^{i}(G)= \begin{cases}G & \text { if } i=0 \\ P_{k}\left(P_{k}^{i-1}(G)\right) & \text { if } i>0\end{cases}
$$

As $P_{1}(G)$ is the usual line graph of $G$, instead of $P_{1}^{i}(G)$ we write $L^{i}(G)$. It is easy to see that the vertex set of the path graph $P_{2}(G)$ is identical to the vertex set of iterated line graph $L^{2}(G)$. However, $P_{2}(G)$ has in general fewer edges than $L^{2}(G)$. For iterated line graphs we have the following analogues of Theorem 1 and Theorem 2, see [8, Theorem 1] and [8, Theorem 2], respectively. 
Theorem B. Let $G$ be a connected graph with minimum degree $\delta \geq 3$. Then $L^{2}(G)$ is ( $\left.\delta-1\right)$-connected.

Theorem C. Let $G$ be a 4-connected graph. Then $L^{2}(G)$ is $(4 \delta-6)$-connected.

We recall that if $G$ is $\delta$-regular graph, then $L^{2}(G)$ is $(4 \delta-6)$-regular. Finally, by [8, Theorem 3] we have:

Theorem D. Let $G$ be a connected $\delta$-regular graph, $\delta \geq 3$. Then for all $i, i \geq 5$, the connectivity of $L^{i}(G)$ equals the degree of $L^{i}(G)$.

Hence, although the degree of $L^{i}(G)$ grows exponentially as a function of $i$, the connectivity of $L^{i}(G)$ attains its theoretical maximum.

For $P_{2}$-path graphs we have the analogous result. As a straightforward consequence of Theorem 1 and Theorem 2 we have:

Theorem 3. Let $G$ be a connected $\delta$-regular graph, $\delta \geq 3$. Then for all $i, i \geq 2$, the connectivity of $P_{2}^{i}(G)$ equals the degree of $P_{2}^{i}(G)$.

We remark that if $G$ is a connected $\delta$-regular graph, $\delta \geq 3$, then the degree of $P_{2}^{i}(G)$ equals the degree of $L^{i}(G)$. Hence, the degree of $P_{2}^{i}(G)$ grows exponentially as a function of $i$.

All proofs and necessary notions are postponed to the next section.

\section{Proofs}

We use standard graph-theoretic notation. Let $G$ be a graph. The vertex set and the edge set of $G$, respectively, are denoted by $V(G)$ and $E(G)$. For two subgraphs, $H_{1}$ and $H_{2}$ of $G$, we denote by $H_{1} \cup H_{2}$ the union of $H_{1}$ and $H_{2}$. If $u$ is a vertex of $G$, then $\operatorname{deg}_{G}(u)$ denotes the degree of $u$ in $G$.

Throughout the paper we use the following definition of vertex-connectivity:

Definition. A graph $G$ is $k$-vertex-connected (or simply $k$-connected) if and only if it has at least $k+1$ vertices, and if for every pair $u$ and $v$ of non-adjacent vertices of $G$ there are at least $k$ internally-vertex-disjoint $u-v$ paths in $G$.

We will have occasion to use the following equivalent formulations (see e.g. [4]).

(i) If $G$ is $k$-connected graph, then for every pair of sets of its vertices $U$ and $V$ such that $|U|=|V|=l \geq k$, there are $k$ vertex-disjoint paths connecting $k$ vertices of $U$ with $k$ vertices of $V$.

(ii) If $G$ is $k$-connected graph, then excluding $l<k$ vertices from $G$ will result in $(k-l)$-connected graph.

To simplify the notation we adopt the following convention. We denote the vertices of $P_{2}(G)$ (as well as the vertices of $G$ ) by small letters $u, v, \ldots$, while the corresponding paths of length 2 will be denoted by capital letters $U, V, \ldots$ This means that if $U$ is a path of length 2 in $G$ and $u$ is a vertex in $P_{2}(G)$, then $U$ must be the path corresponding to the vertex $u$. In some places, when no confusion is expected, we replace a vertex of $P_{2}(G)$ by the corresponding path. To avoid any misunderstandings, we denote a vertex of $P_{2}(G)$ (i.e., a path of length 2 in $G$ ) as a 
triple of vertices in parentheses, say $U=\left(u_{0}, u_{1}, u_{2}\right)$, where the middle vertex $\left(u_{1}\right.$ in this case) has degree 2 in $U$; while the other paths in $G$ (and also the paths in $P_{2}(G)$ ), we denote without parentheses. That is to say, we denote by $P=v_{1}, v_{2}, v_{3}$ a path of length 2 starting in $v_{1}$, passing through $v_{2}$, and terminating in $v_{3}$. This enables us to write an extension of $P$, by $v_{0}$ in the beginning and by $v_{4}$ at the end, as $v_{0}, P, v_{4}$.

We introduce some terminology which will be useful in proving our results.

Let $G$ be a graph with minimum degree $\delta \geq 3$. For every vertex $b$ of $G$ we define a cyclic permutation (rotation) $\rho_{b}$ of the neighbours of $b$. If $x$ and $y$ are two neighbours of $b$, and $y$ is the image of $x$ in the rotation $\rho_{b}$, we write $y=\rho_{b}(x)$.

Let $P^{\prime}=a_{1}, a_{2}, \ldots, a_{t-1}$ be a nonempty path in $G$ without chords, i.e., whenever $a_{i} a_{j} \in E(G), 1 \leq i, j \leq t-1$, then $|i-j|=1$. Let $a_{0}$ be a neighbour of $a_{1}$ such that $a_{0} \notin V\left(P^{\prime}\right)$, and let $a_{t}$ be a neighbour of $a_{t-1}$ such that $a_{t} \notin V\left(P^{\prime}\right)$. Denote $P=a_{0}, P^{\prime}, a_{t}$. Then $P$ is either a path or a cycle, and its length is at least two.

For every $i, 1 \leq i \leq t-1$, by $b_{i, j}$ we denote $\delta-2$ distinct neighbours of $a_{i}$, $1 \leq j \leq \delta-2$, which are different from $a_{i-1}$ and $a_{i+1}$. Moreover, let $c_{i, j}$ be a neighbour of $b_{i, j}$ such that $c_{i, j}=\rho_{b_{i, j}}\left(a_{i}\right)$. Then $P$-based paths are the following walks in $P_{2}(G)$ :

(1) For every $j, 1 \leq j \leq \delta-2$, the $j$-th path based on $P$ is $\left(a_{0}, a_{1}, b_{1, j}\right)$, $\left(a_{1}, b_{1, j}, c_{1, j}\right),\left(b_{1, j}, a_{1}, a_{2}\right),\left(a_{1}, a_{2}, b_{2, j}\right),\left(a_{2}, b_{2, j}, c_{2, j}\right),\left(b_{2, j}, a_{2}, a_{3}\right), \ldots$, $\left(a_{t-1}, b_{t-1, j}, c_{t-1, j}\right),\left(b_{t-1, j}, a_{t-1}, a_{t}\right)$.

(2) A straight path based on $P$ is $\left(a_{0}, a_{1}, a_{2}\right),\left(a_{1}, a_{2}, a_{3}\right), \ldots,\left(a_{t-2}, a_{t-1}, a_{t}\right)$.

The collection of $P$-based paths defined above is a bunch of $P$-based paths, and we denote this collection by $B(P)$. Since all edges $a_{i} b_{i, j}$ are mutually distinct, also the edges $b_{i, j} c_{i, j}$ are mutually distinct. Consequently, although we may have $c_{i, j}=a_{i^{*}}$ for some $i, j$ and $i^{*}$, as $\delta \geq 3$ all the paths $a_{i}, b_{i, j}, c_{i, j}$ are mutually distinct. Hence, $B(P)$ forms a collection of vertex-disjoint paths in $P_{2}(G)$.

Proof of Theorem 1. Let $u$ and $v$ be non-adjacent vertices of $P_{2}(G), U=\left(u_{0}, u_{1}, u_{2}\right)$ and $V=\left(v_{0}, v_{1}, v_{2}\right)$. We construct $\delta-1$ internally-vertex-disjoint $u-v$ paths in $P_{2}(G)$.

If $U$ and $V$ share an edge in common, we may assume that $u_{0}=v_{0}$ and $u_{1}=v_{1}$, as $u$ and $v$ are not adjacent. Since $\operatorname{deg}_{G}\left(u_{0}\right) \geq \delta$, there are $\delta-1$ distinct vertices in $P_{2}(G)$ of the form $\left(u_{1}, u_{0}, x\right), x \in V(G)$, and all these vertices are the middle vertices of paths of length 2 joining $u$ with $v$. Hence, if $U$ and $V$ are not edgedisjoint, then there are $\delta-1$ internally-vertex-disjoint $u-v$ paths in $P_{2}(G)$.

Now suppose that $U$ and $V$ are edge-disjoint. By symmetry, there are three cases to distinguish:

(1) There is a path $P^{\prime}$ in $G$ connecting an endvertex of $U$ with an endvertex of $V$, and which avoids both $u_{1}$ and $v_{1}$. Moreover, let $P^{\prime}$ be a shortest path of this type. Then $P^{\prime}$ has no chords. Let $P=u_{1}, P^{\prime}, v_{1}$. Then $P$ is a path or a cycle, and its length is at least two. Hence, there is a bunch of $P$-based paths $B(P)$, and the paths of $B(P)$ form a collection of $\delta-1$ vertex-disjoint paths in $P_{2}(G)$. Now as all first vertices of these paths are adjacent to $u$ and all endvertices are adjacent to $v$, there are $\delta-1$ internally-vertex-disjoint $u-v$ paths in $P_{2}(G)$. 
(2) There is a path $P^{\prime}$ in $G$ connecting $u_{1}$ with $v_{0}$ and avoiding $v_{1}$, but there is no path of type (1). Moreover, let $P^{\prime}$ be a shortest path of this type. Then $P^{\prime}$ has no chords. Let $P=u_{2}, P^{\prime}, v_{1}$. Then $P$ is a path and its length is at least two. (Recall that there is no path of type (1), and $P^{\prime}$ is a shortest path of type (2) in $G$.) All endvertices of vertex-disjoint paths in $B(P)$ are adjacent to $v$, and every first vertex is adjacent to all vertices $\left(u_{1}, u_{2}, x\right)$, where $x$ is a neighbour of $u_{2}, x \neq u_{1}$. As there are $\delta-1$ vertices $\left(u_{1}, u_{2}, x\right)$, all these vertices are adjacent to $u$, and (as $u_{2}$ is nonadjacent to a vertex of $P^{\prime}$ distinct from $u_{1}$ ) they are distinct from the vertices in $B(P)$; the paths in $B(P)$ can be extended to $\delta-1$ internally-vertex-disjoint $u-v$ paths in $P_{2}(G)$.

(3) There is a path $P^{\prime}$ in $G$ connecting $u_{1}$ with $v_{1}$, but there are no paths of types (1) and (2). This case is similar to case (2), but the construction has to be applied in both ends of the bunch of $P$-based paths.

In the proof of Theorem 2 we use two "modified" bunches of paths to obtain $2 \delta-2$ vertex-disjoint paths. Let $P^{1}=a_{0}^{1}, a_{1}^{1}, \ldots, a_{t^{1}}^{1}$ and $P^{2}=a_{0}^{2}, a_{1}^{2}, \ldots, a_{t^{2}}^{2}$ be paths or cycles, each of length at least 2 , such that their subpaths $P^{1^{\prime}}=a_{1}^{1}, a_{2}^{1}, \ldots, a_{t^{1}-1}^{1}$ and $P^{2 \prime}=a_{1}^{2}, a_{2}^{2}, \ldots, a_{t^{2}-1}^{2}$ are vertex-disjoint and chordless. Moreover, let $a_{0}^{1}, a_{t^{1}}^{1} \notin$ $V\left(P^{2 \prime}\right)$ and $a_{0}^{2}, a_{t^{2}}^{2} \notin V\left(P^{1 \prime}\right)$. Let $\mathcal{P}^{1}=B\left(P^{1}\right)$ and $\mathcal{P}^{2}=B\left(P^{2}\right)$. As mentioned above, both $\mathcal{P}^{1}$ and $\mathcal{P}^{2}$ are collections of vertex-disjoint paths, but the paths in $\mathcal{P}^{1} \cup \mathcal{P}^{2}$ are not necessarily vertex-disjoint. By the rotations, no problem occurs if there is a vertex $b \in V(G)$ with at least two neighbours in $V\left(P^{1 \prime} \cup P^{2 \prime}\right)$, such that $b \notin V\left(P^{1 \prime} \cup P^{2 \prime}\right)$. Complications arise if there is an edge $a_{i^{1}}^{1} a_{i^{2}}^{2}$ in $G$ such that $1 \leq i^{1} \leq t^{1}-1$ and $1 \leq i^{2} \leq t^{2}-1$.

To obtain a collection of $2 \delta-2$ vertex-disjoint paths from $\mathcal{P}^{1} \cup \mathcal{P}^{2}$, we check step by step all the edges $a_{i^{1}}^{1} a_{i^{2}}^{2}, 1 \leq i^{1} \leq t^{1}-1$ and $1 \leq i^{2} \leq t^{2}-1$, such that the edge $a_{i^{1}}^{1} a_{i^{2}}^{2}$ is in a vertex of $\mathcal{P}^{1} \cup \mathcal{P}^{2}$. Let $\mathcal{P}=\mathcal{P}^{1} \cup \mathcal{P}^{2}$. Observe that if a path in $\mathcal{P}^{1}$ contains $\left(a_{i^{1}-1}^{1}, a_{i^{1}}^{1}, a_{i^{2}}^{2}\right)$, then it contains also $\left(a_{i^{2}}^{2}, a_{i^{1}}^{1}, a_{i^{1}+1}^{1}\right)$; and similarly, if a path in $\mathcal{P}^{2}$ contains $\left(a_{i^{2}-1}^{2}, a_{i^{2}}^{2}, a_{i^{1}}^{1}\right)$, then it contains also $\left(a_{i^{1}}^{1}, a_{i^{2}}^{2}, a_{i^{2}+1}^{2}\right)$. We proceed according to the following rules:

(i) Suppose that there is a path $Q_{1}$ in $\mathcal{P}$ containing a subpath $\left(a_{i^{1}-1}^{1}, a_{i^{1}}^{1}, a_{i^{2}}^{2}\right)$, $\left(a_{i^{1}}^{1}, a_{i^{2}}^{2}, c_{1}\right),\left(a_{i^{2}}^{2}, a_{i^{1}}^{1}, a_{i^{1}+1}^{1}\right)$ for some $c_{1}$, and there is also a path $Q_{2}$ in $\mathcal{P}$ containing a subpath $\left(a_{i^{2}-1}^{2}, a_{i^{2}}^{2}, a_{i^{1}}^{1}\right),\left(a_{i^{2}}^{2}, a_{i^{1}}^{1}, c_{2}\right),\left(a_{i^{1}}^{1}, a_{i^{2}}^{2}, a_{i^{2}+1}^{2}\right)$ for some $c_{2}$. There are two cases to distinguish.

If $Q_{1} \neq Q_{2}$, we construct two new paths $Q_{1}^{*}$ and $Q_{2}^{*}$ :

$Q_{1}^{*}=$ first part of $Q_{1},\left(a_{i^{1}-1}^{1}, a_{i^{1}}^{1}, a_{i^{2}}^{2}\right),\left(a_{i^{1}}^{1}, a_{i^{2}}^{2}, a_{i^{2}+1}^{2}\right)$, second part of $Q_{2}$ ； $Q_{2}^{*}=$ first part of $Q_{2},\left(a_{i^{2}-1}^{2}, a_{i^{2}}^{2}, a_{i^{1}}^{1}\right),\left(a_{i^{2}}^{2}, a_{i^{1}}^{1}, a_{i^{1}+1}^{1}\right)$, second part of $Q_{1}$.

Denote by $\mathcal{P}$ the collection $\left(\mathcal{P}-\left\{Q_{1}, Q_{2}\right\}\right) \cup\left\{Q_{1}^{*}, Q_{2}^{*}\right\}$. Then $V\left(Q_{1}^{*} \cup Q_{2}^{*}\right) \subset$ $V\left(Q_{1} \cup Q_{2}\right)$, and there is no conflict on the edge $a_{i^{1}}^{1} a_{i^{2}}^{2}$ in $\mathcal{P}$.

If $Q_{1}=Q_{2}$, we construct one new path $Q_{1}^{*}$. Suppose that $\left(a_{i^{1}-1}^{1}, a_{i^{1}}^{1}, a_{i^{2}}^{2}\right)$ preceeds $\left(a_{i^{2}-1}^{2}, a_{i^{2}}^{2}, a_{i^{1}}^{1}\right)$ on $Q_{1}$. The other subcase can be resolved similarly. Then

$Q_{1}^{*}=$ first part of $Q_{1},\left(a_{i^{1}-1}^{1}, a_{i^{1}}^{1}, a_{i^{2}}^{2}\right),\left(a_{i^{1}}^{1}, a_{i^{2}}^{2}, a_{i^{2}+1}^{2}\right)$, terminal part of $Q_{1}$. 
Denote by $\mathcal{P}$ the collection $\left(\mathcal{P}-\left\{Q_{1}\right\}\right) \cup\left\{Q_{1}^{*}\right\}$. As above, there is no conflict on the edge $a_{i^{1}}^{1} a_{i^{2}}^{2}$ in $\mathcal{P}$.

(ii) Suppose that there is a path $Q_{1}$ in $\mathcal{P}$ containing the subpath $\left(a_{i^{1}-1}^{1}, a_{i^{1}}^{1}, a_{i^{2}}^{2}\right)$, $\left(a_{i^{1}}^{1}, a_{i^{2}}^{2}, c_{1}\right),\left(a_{i^{2}}^{2}, a_{i^{1}}^{1}, a_{i^{1}+1}^{1}\right)$ for some $c_{1}$, but no path in $\mathcal{P}$ contains the subpath $\left(a_{i^{2}-1}^{2}, a_{i^{2}}^{2}, a_{i^{1}}^{1}\right),\left(a_{i^{2}}^{2}, a_{i^{1}}^{1}, c_{2}\right),\left(a_{i^{1}}^{1}, a_{i^{2}}^{2}, a_{i^{2}+1}^{2}\right)$ for any $c_{2}$. Replace $c_{1}$ by $a_{i^{2}-1}^{2}$ in $Q_{1}$, and denote the resulting path by $Q_{1}^{*}$. Further, denote by $\mathcal{P}$ the collection $\left(\mathcal{P}-\left\{Q_{1}\right\}\right) \cup\left\{Q_{1}^{*}\right\}$. Clearly, there is no conflict on the edge $a_{i^{1}}^{1} a_{i^{2}}^{2}$ in $\mathcal{P}$.

(iii) Suppose that there is a path $Q_{2}$ in $\mathcal{P}$ containing the subpath $\left(a_{i^{2}-1}^{2}, a_{i^{2}}^{2}, a_{i^{1}}^{1}\right)$, $\left(a_{i^{2}}^{2}, a_{i^{1}}^{1}, c_{2}\right),\left(a_{i^{1}}^{1}, a_{i^{2}}^{2}, a_{i^{2}+1}^{2}\right)$ for some $c_{2}$, but no path in $\mathcal{P}$ contains the subpath $\left(a_{i^{1}-1}^{1}, a_{i^{1}}^{1}, a_{i^{2}}^{2}\right),\left(a_{i^{1}}^{1}, a_{i^{2}}^{2}, c_{1}\right),\left(a_{i^{2}}^{2}, a_{i^{1}}^{1}, a_{i^{1}+1}^{1}\right)$ for any $c_{1}$. Replace $c_{2}$ by $a_{i^{1}-1}^{1}$ in $Q_{2}$, and denote the resulting path by $Q_{2}^{*}$. Further, denote by $\mathcal{P}$ the collection $\left(\mathcal{P}-\left\{Q_{2}\right\}\right) \cup\left\{Q_{2}^{*}\right\}$. As above, there is no conflict on the edge $a_{i^{1}}^{1} a_{i^{2}}^{2}$ in $\mathcal{P}$.

When all edges $a_{i^{1}}^{1} a_{i^{2}}^{2}$, such that $1 \leq i^{1} \leq t^{1}-1$ and $1 \leq i^{2} \leq t^{2}-1$, are checked, the resulting collection $\mathcal{P}$ of paths is called a modified bunch of $P^{1}, P^{2}$-based paths, and it is denoted by $M B\left(\mathcal{P}^{1}, \mathcal{P}^{2}\right)$. By construction, $M B\left(\mathcal{P}^{1}, \mathcal{P}^{2}\right)$ is a collection of vertex-disjoint paths.

We remark that $M B(X, Y)$ is defined similarly for $X \subseteq B\left(P^{1}\right)$ and $Y \subseteq B\left(P^{2}\right)$. In fact, we use this notation also in the case when one path of $B\left(P^{1}\right)$, say $Q_{1}$, is replaced by a path $Q_{1}^{*}$; such that $Q_{1}^{*}$ consists of a straight path based on $P^{3}\left(P^{3}\right.$ contains no vertex of $P^{2}$, and only the last vertex of $P^{3}$ is in $\left.P^{1}\right)$, then it continues with a subpath of $Q_{1}$, and it terminates with a straight path based on $P^{4}\left(P^{4}\right.$ contains no vertex of $P^{2}$, and only the first vertex of $P^{4}$ is in $\left.P^{1}\right)$. But in this case we write $M B\left(\left(\mathcal{P}^{1}-\left\{Q_{1}\right\}\right) \cup Q_{1}^{*}, \mathcal{P}^{2}\right)$ to indicate the change.

Further, we remark that if $P^{1}$ and $P^{2}$ are paths as described above with exactly one change, namely that $a_{0}^{1}=a_{1}^{2}$, then $M B\left(\mathcal{P}^{1}, \mathcal{P}^{2}\right)$ is not necessarily a collection of vertex-disjoint paths in $P_{2}(G)$ (here $\mathcal{P}^{1}=B\left(P^{1}\right)$ and $\mathcal{P}^{2}=B\left(P^{2}\right)$ ). However, if we restrict ourselves to subpaths of bunch of $P^{1}$-based paths, i.e., if $P^{3}$ is defined by $P^{1}=a_{0}^{1}, P^{3}$ and $\mathcal{P}^{3}=B\left(P^{3}\right)$, then $M B\left(\mathcal{P}^{3}, \mathcal{P}^{2}\right)$ is a collection of vertex-disjoint paths. Hence, possible complications occur only in the first two vertices of paths of $\mathcal{P}^{1}$.

Proof of Theorem 2. Let $u$ and $v$ be non-adjacent vertices in $P_{2}(G), U=\left(u_{0}, u_{1}, u_{2}\right)$ and $V=\left(v_{0}, v_{1}, v_{2}\right)$. We construct $2 \delta-2$ internally-vertex-disjoint $u-v$ paths in $P_{2}(G)$. We remark that if $\operatorname{deg}_{G}\left(u_{0}\right)=\operatorname{deg}_{G}\left(u_{2}\right)=\delta$, then $\operatorname{deg}_{P_{2}(G)}(u)=2 \delta-2$. Hence, $\delta-1$ of our $u-v$ paths must leave the vertex $u$ via $\left(u_{1}, u_{0}, x\right), x \in V(G)$, and $\delta-1$ of them must pass through $\left(u_{1}, u_{2}, y\right), y \in V(G)$. Analogously, $\delta-1$ of the paths must pass through $\left(x, v_{0}, v_{1}\right), x \in V(G)$, and $\delta-1$ of them must contain $\left(y, v_{2}, v_{1}\right), y \in V(G)$.

First suppose that $U$ and $V$ share an edge in common, say $u_{0}=v_{0}$ and $u_{1}=v_{1}$. As shown in the proof of Theorem 1, there is a collection $\mathcal{T}$ of $\delta-1$ internally-vertexdisjoint $u-v$ paths of length 2 in $P_{2}(G)$. Further, as $G$ is 2-connected graph, there is a $u_{2}-v_{2}$ path $P^{1 \prime}=u_{2}, a_{2}^{1}, a_{3}^{1}, \ldots, a_{t^{1}-2}^{1}, v_{2}$ in $G-\left\{u_{1}\right\}$. Let $P^{1}=u_{1}, P^{1 \prime}, u_{1}$, 
and let $Q_{1}$ be the straight path based on $P^{1}$. Clearly, $Q_{1}$ contains no vertex of $\mathcal{T}$, even if $P^{1}$ passes through $u_{0}$.

Let $P^{2}=u_{2}, u_{1}, v_{2}$, and let $\mathcal{P}^{2}=B\left(P^{2}\right)$. In $\mathcal{P}^{2}$ there is at most one path, say $Q_{2}$, containing the vertex $\left(u_{2}, u_{1}, u_{0}\right)$. All first vertices of paths in $\mathcal{P}^{2}-\left\{Q_{2}\right\}$ are adjacent to $\delta-2$ neighbours of $u$ of the form $\left(u_{1}, u_{2}, x\right), x \neq a_{2}^{1}$, and all terminal vertices of paths in $\mathcal{P}^{2}-\left\{Q_{2}\right\}$ are adjacent to neighbours of $v$ of the form $\left(u_{1}, v_{2}, x\right), x \neq a_{t^{1}-2}^{1}$. Hence, the paths of $\left(\mathcal{P}^{2}-\left\{Q_{2}\right\}\right) \cup\left\{Q_{1}\right\}$ can be extended to $\delta-1$ internally-vertex-disjoint $u-v$ paths, and these paths together with the paths of $\mathcal{T}$ form a collection of $2 \delta-2$ internally-vertex-disjoint $u-v$ paths in $P_{2}(G)$, see Figure 1 (the numbers 1 and $\delta-2$ denote how many $P^{1}$-based and $P^{2}$-based paths of $B\left(P^{1}\right)$ and $B\left(P^{2}\right)$, respectively, are used).

Now suppose that $U$ and $V$ are edge-disjoint. As $G$ is 2-connected, there are two vertex-disjoint paths, say $P^{1 \prime}$ and $P^{2 \prime}$, connecting distinct vertices of $U$ with distinct vertices of $V$. By symmetry, there are four cases to distinguish:

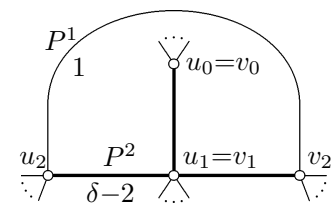

Figure 1

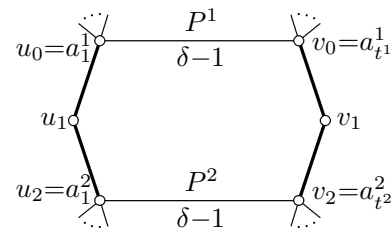

Figure 2

(1) $P^{1 \prime}$ connects $u_{0}$ with $v_{0}$ and $P^{2 \prime}$ connects $u_{2}$ with $v_{2}$. Assume that both $P^{1 \prime}$ and $P^{2 \prime}$ are as short as possible. Let $P^{1}=u_{1}, P^{1 \prime}, v_{1}$ and $P^{2}=u_{1}, P^{2 \prime}, v_{1}$. Then each of $P^{1}$ and $P^{2}$ has length at least 2. Denote $\mathcal{P}^{1}=B\left(P^{1}\right)$ and $\mathcal{P}^{2}=B\left(P^{2}\right)$. Then $M B\left(\mathcal{P}^{1}, \mathcal{P}^{2}\right)$ is a collection of $2 \delta-2$ vertex-disjoint paths, such that all first vertices of these paths are adjacent to $u$, and their endvertices are adjacent to $v$. Hence, there is a collection of $2 \delta-2$ internally-vertex-disjoint $u-v$ paths in $P_{2}(G)$, see Figure 2.

(2) $P^{1 \prime}$ connects $u_{0}$ with $v_{0}$ and $P^{2 \prime}$ connects $u_{1}$ with $v_{2}$. Assume that both $P^{1 \prime}$ and $P^{2 \prime}$ are as short as possible, and moreover, assume that there are no paths of type (1) in $G$. Let $P^{1}=u_{1}, P^{1 \prime}, v_{1}$ and $P^{2}=u_{2}, P^{2 \prime}, v_{1}$. As usual, we denote $P^{1}=a_{0}^{1}, a_{1}^{1}, \ldots, a_{t^{1}}^{1}$ and $P^{2}=a_{0}^{2}, a_{1}^{2}, \ldots, a_{t^{2}}^{2}$. If $\operatorname{deg}_{G}\left(u_{1}\right)=\operatorname{deg}_{G}\left(u_{0}\right)=\delta$, then the paths in any modified bunch of $P^{1}, P^{2}$-based paths are not vertex-disjoint. As mentioned above, complications may occur only in the first two vertices of the paths, and in fact, only the vertex $\left(u_{1}, u_{0}, x\right)$ appears twice in the paths of the modified bunch of $P^{1}, P^{2}$-based paths, for suitable $x$. However, as $G$ is 2connected, there is a path $P^{3}=a_{0}^{3}, a_{1}^{3}, \ldots, a_{t^{3}}^{3}$ in $G-\left\{u_{1}\right\}$ connecting $u_{2}$ with a vertex of $P^{1} \cup P^{2}-\left\{u_{1}, u_{2}\right\}$. Since there are no paths of type (1) in $G, P^{3}$ joins $u_{2}$ with a vertex $a_{p}^{1}$ of $P^{1}$, see Figure 3 (by dashed lines the range of terminal vertices of $P^{3}$ is denoted). Assume that $P^{3}$ is as short as possible, and then we assume that the index $p$ is as large as possible. 


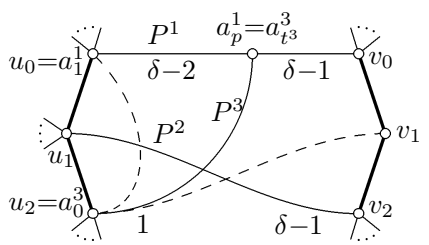

Figure 3

Now we define some rotations and labellings, so that the paths of the modified bunch of $P^{1}, P^{2}$-based paths will fit together with the straight path based on $P^{3}$. If $P^{3}$ terminates in $u_{0}$, i.e. if $a_{t^{3}}^{3}=a_{p}^{1}=u_{0}$, then we relabel the rotation in $u_{0}$ so that $\rho_{u_{0}}\left(u_{1}\right)=a_{t^{3}-1}^{3}$. Since $u_{0}$ is adjacent to an interior vertex $u_{1}=a_{1}^{2}$ of $P^{2}$, there is $j^{*}, 1 \leq j^{*} \leq \delta-2$, such that $b_{1, j^{*}}^{2}=u_{0}$. Then our rotation forces that $c_{1, j^{*}}^{2}=a_{t^{3}-1}^{3}$ if $a_{t^{3}}^{3}=u_{0}$. Further, relabel the $b_{i, j}^{1}$ 's and $c_{i, j}^{1}$ 's so that $b_{1,1}^{1}=c_{1, j^{*}}^{2}$ and $b_{p, 1}^{1}=a_{t^{3}-1}^{3}$. Moreover, for all edges $a_{i}^{1} u_{2} \in E(G)$ (by our assumptions there are at most two such edges, namely $a_{1}^{1} u_{2}$ and $a_{2}^{1} u_{2}$ ) we choose $b_{i, 1}^{1}=u_{2}$. As $P^{3}$ is as short as possible, all these relabellings are consistent.

Let $\mathcal{P}^{1}=B\left(P^{1}\right), \mathcal{P}^{2}=B\left(P^{2}\right)$, and let $Q$ be the first path based on $P^{1}$. Denote

$$
\begin{aligned}
Q^{*}= & \left(u_{1}, u_{2}, a_{1}^{3}\right), \text { straight path based on } P^{3},\left(a_{t^{3}-1}^{3}, a_{p}^{1}, a_{p+1}^{1}\right), \\
& \left(a_{p}^{1}, a_{p+1}^{1}, b_{p+1,1}^{1}\right), \text { terminal subpath of } Q .
\end{aligned}
$$

We remark that if $a_{t^{3}}^{3}=v_{1}$, then there is no terminal subpath of $Q$ in $Q^{*}$.

It is a matter of routine to check that $M B\left(\left(\mathcal{P}^{1}-\{Q\}\right) \cup Q^{*}, \mathcal{P}^{2}\right)$ is a collection of $2 \delta-2$ vertex-disjoint paths. Some first (terminal) vertices of these paths are already adjacent to $u$ (to $v$ ), but some of the paths must be enlarged. Exactly $\delta-1$ of these paths start with vertices $\left(u_{2}, u_{1}, x\right), x \in V(G)$. One such path starts already in $u$; but the remaining $\delta-2$ we extend, each by one vertex of the form $\left(y, u_{2}, u_{1}\right), y \neq a_{1}^{3}$, that is already adjacent to $u$. By the choice of $b_{1, j}^{1}$ 's of $Q$, no vertex $\left(y, u_{2}, u_{1}\right), y \neq a_{1}^{3}$, appears in $M B\left(\left(\mathcal{P}^{1}-\{Q\}\right) \cup Q^{*}, \mathcal{P}^{2}\right)$.

Hence, it remains to extend $Q^{*}$ at the end in the case $a_{p}^{1}=v_{1}$. In this case we enlarge $Q^{*}$ by subpath $\left(a_{t^{3}-1}^{3}, a_{p}^{1}, a_{p-1}^{1}\right),\left(a_{p}^{1}, a_{p-1}^{1}, b_{p-1,1}^{1}\right), v$, where $a_{p}^{1}=a_{t^{3}}^{3}=v_{1}$ and $a_{p-1}^{1}=v_{0}$. We remark that if $b_{p-1,1}^{1}$ is an interior vertex of $P^{2}$, say $b_{p-1,1}^{1}=a_{r}^{2}$ and $a_{p-1}^{1}=b_{r, j^{\prime}}^{2}$, then choosing $c_{r, j^{\prime}}^{2}=a_{p-2}^{1}$ guarantees that all these extended paths are vertex-disjoint. Hence, there is a collection of $2 \delta-2$ internally-vertexdisjoint $u-v$ paths in $P_{2}(G)$.

(3) $P^{1 \prime}$ connects $u_{0}$ with $v_{0}$ and $P^{2 \prime}$ connects $u_{1}$ with $v_{1}$. Assume that both $P^{1 \prime}$ and $P^{2 \prime}$ are as short as possible, and moreover, assume that there are no paths of types (1) and (2) in $G$. Let $P^{1}=u_{1}, P^{1 \prime}, v_{1}$ and $P^{2}=u_{2}, P^{2 \prime}, v_{2}$. As usual, we denote $P^{1}=a_{0}^{1}, a_{1}^{1}, \ldots, a_{t^{1}}^{1}$ and $P^{2}=a_{0}^{2}, a_{1}^{2}, \ldots, a_{t^{2}}^{2}$. As $G$ is 2-connected graph, there is a path $P^{3 \prime}$ in $G-\left\{u_{1}\right\}$ connecting $u_{2}$ with a vertex of $P^{1} \cup P^{2}-\left\{u_{1}, u_{2}\right\}$. Since there are no paths of types (1) and (2) in $G, P^{3 \prime}$ joins $u_{2}$ with a vertex $a_{p_{3}}^{1}$ of $P^{1^{\prime}}$. Analogously, there is a path $P^{4 \prime}$ joining a vertex of $P^{1 \prime}$, say $a_{p_{4}}^{1}$, 
with $v_{2}$. Assume that both $P^{3 \prime}$ and $P^{4 \prime}$ are as short as possible, and denote $P^{3}=u_{1}, P^{3 \prime}=a_{0}^{3}, a_{1}^{3}, \ldots, a_{t^{3}}^{3}$ and $P^{4}=P^{4 \prime}, v_{1}=a_{0}^{4}, a_{1}^{4}, \ldots, a_{t^{4}}^{4}$, see Figure 4.

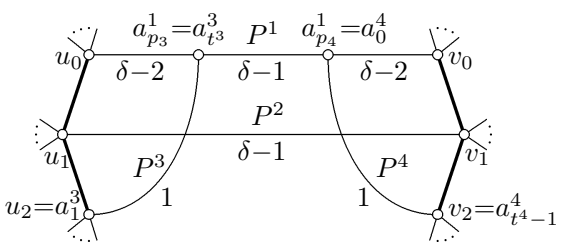

Figure 4

In $a_{t^{3}-1}^{3}$ we choose the rotation so that $\rho_{a_{t^{3}-1}^{3}}\left(a_{t^{3}-2}^{3}\right)=a_{t^{3}}^{3}=a_{p_{3}}^{1}$ (hence $\left.\rho_{a_{t^{3}-1}^{3}}\left(a_{t^{3}}^{3}\right) \neq a_{t^{3}-2}^{3}\right)$, and in $a_{1}^{4}$ we require $\rho_{a_{1}^{4}}\left(a_{2}^{4}\right)=a_{0}^{4}$. If $a_{p_{3}}^{1} \neq a_{p_{4}}^{1}$, then we choose $b_{p_{3}, 1}^{1}=a_{t^{3}-1}^{3}$ and $b_{p_{4}, 1}^{1}=a_{1}^{4}$. By our assumptions, if $u_{2} a_{j}^{1} \in E(G), j>0$, then $j=1$ or $j=2$. Hence, at most two interior vertices of $P^{1}$ are adjacent to $u_{2}$. If there is just one interior vertex of $P^{1}$ adjacent to $u_{2}$, then by the previous choice we have $\rho_{a^{3}-1}^{3}\left(a_{t^{3}}^{3}\right)=\rho_{u_{2}}\left(a_{p_{3}}^{1}\right) \neq u_{1}$. On the other hand if $u_{2} a_{1}^{1}, u_{2} a_{2}^{1} \in E(G)$, then we choose either $b_{1,1}^{1}=u_{2}$ or $b_{2,1}^{1}=u_{2}$ (not to abuse the list of the requirements). In the last case suppose that $b_{1,1}^{1}=u_{2}$ and $b_{2,1}^{1} \neq u_{2}$. Then there is $j^{*}$ such that $b_{2, j^{*}}^{1}=u_{2}$, and we choose (although not corresponding to the rotations) $c_{2, j^{*}}^{1}=a_{1}^{1}$. (The subcase $b_{1,1}^{1} \neq u_{2}$ and $b_{2,1}^{1}=u_{2}$ can be solved similarly.) Analogously we proceed in the case when an interior vertex of $P^{1}$ is adjacent to $v_{2}$.

Let $\mathcal{P}^{1}=B\left(P^{1}\right), \mathcal{P}^{2}=B\left(P^{2}\right)$, and let $Q$ be the first path based on $P^{1}$. If $a_{p_{3}}^{1}=a_{p_{4}}^{1}$ then we denote

$$
\begin{aligned}
Q^{*}= & \left(u_{1}, u_{2}, a_{2}^{3}\right), \text { straight path based on } P^{3},\left(a_{t^{3}-1}^{3}, a_{p_{3}}^{1}, a_{1}^{4}\right), \\
& \text { straight path based on } P^{4},\left(a_{t^{4}-2}^{4}, v_{2}, v_{1}\right),
\end{aligned}
$$

and if $a_{p_{3}}^{1} \neq a_{p_{4}}^{1}$ then two changes are necessary. If $\left|p_{3}-p_{4}\right|>1$, we change the straight path based on $P^{1}$ by replacing the piece between $a_{p_{3}}^{1}$ and $p_{p_{4}}^{1}$ by the corresponding subpath of $Q$, and we denote

$$
\begin{aligned}
Q^{*}= & \left(u_{1}, u_{2}, a_{2}^{3}\right), \text { straight path based on } P^{3}, \bar{u}, \text { subpath of the straight } \\
& \text { path based on } P^{1}, \bar{v}, \text { straight path based on } P^{4},\left(a_{t^{4}-2}^{4}, v_{2}, v_{1}\right),
\end{aligned}
$$

where the subpath of the straight path based on $P^{1}$ is in a reverse order if $p_{3}>p_{4}$, $\bar{U}=\left(a_{t^{3}-1}^{3}, a_{p_{3}}^{1}, a_{p_{3}+1}^{1}\right)$ and $\bar{V}=\left(a_{p_{4}-1}^{1}, a_{p_{4}}^{1}, a_{1}^{4}\right)$ if $p_{3}<p_{4}$, and if $p_{3}>p_{4}$ then $\bar{U}=\left(a_{t^{3}-1}^{3}, a_{p_{3}}^{1}, a_{p_{3}-1}^{1}\right)$ and $\bar{V}=\left(a_{p_{4}+1}^{1}, a_{p_{4}}^{1}, a_{1}^{4}\right)$.

Now $M B\left(\left(\mathcal{P}^{1}-\{Q\}\right) \cup Q^{*}, \mathcal{P}^{2}\right)$ is a collection of $2 \delta-2$ vertex-disjoint paths, and similarly as in case (2) these paths can be extended to $2 \delta-2$ internally-vertexdisjoint $u-v$ paths. 


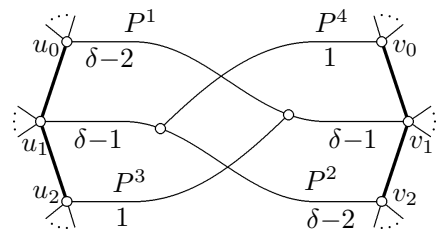

Figure 5

(4) $P^{1 \prime}$ connects $u_{0}$ with $v_{1}$ and $P^{2 \prime}$ connects $u_{1}$ with $v_{2}$. Setting $P^{1}=u_{1}, P^{1 \prime}, v_{0}$ and $P^{2}=u_{2}, P^{2 \prime}, v_{1}$, this case is similar to case (2), but slight changes have to be made in both ends of the modified bunch of $P^{1}, P^{2}$-based paths, see Figure 5 .

\section{REFERENCES}

[1] Aldred R. E. L., Ellingham M. N., Hemminger R. L., Jipsen P., $P_{3}$-isomorphisms for graphs, J. Graph Theory 24 (1997), 35-51.

[2] Belan A., Jurica P., Diameter in path graphs, Acta Math. Univ. Comenian. LXVIII (1999), 111-126.

[3] Broersma H. J., Hoede C., Path graphs, J. Graph Theory 13 (1989), 427-444.

[4] Harary F., Graph Theory, Addison-Wesley, London, 1969.

[5] Knor M., Niepel L., Path, trail and walk graphs, Acta Math. Univ. Comenian. LXVIII (1999), 253-256.

[6] Knor M., Niepel L., Centers in path graphs, JCISS 24 (1999), 79-86.

[7] Knor M., Niepel L., Distances in iterated path graphs, Discrete Math. (to appear).

[8] Knor M., Niepel L., Connectivity of iterated line graphs, (submitted).

[9] Li H., Lin Y., On the characterization of path graphs, J. Graph Theory $\mathbf{1 7}$ (1993), 463-466.

[10] Li X., Zhao B., Isomorphisms of $P_{4}$-graphs, Australasian J. of Combinatorics 15 (1997), 135-143.

[11] Yu X., Trees and unicyclic graphs with Hamiltonian path graphs, J. Graph Theory 14 (1990), 705-708.

(Received 5 Jan 2001) 